\title{
Edge Enhanced Direct Visual Odometry
}

Xin Wang ${ }^{1}$

xinwang_cis@pku.edu.cn

Wei Dong ${ }^{1}$

dongwei92@pku.edu.cn

Mingcai Zhou ${ }^{2}$

mingcai.zhou@samsung.com

Renju Li ${ }^{1}$

lirenju@3dscan.com.cn

Hongbin Zha ${ }^{1}$

zha@cis.pku.edu.cn
${ }^{1}$ Key Laboratory of Machine Perception

School of EECS

Peking University

Beijing, China

${ }^{2}$ Advanced Research Lab

Samsung Research Center-Beijing

Beijing, China
In this paper, we propose an RGB-D visual odometry method that both minimizes the photometric error and aligns the edges between frames. The combination of the direct photometric information [1] and the edge features leads to higher tracking accuracy and enables the approach to deal with challenging texture-less scenes. In contrast to traditional line feature based methods [2], we involve all edges rather than only line segments, avoiding the aperture problem and the uncertainty of endpoints. Instead of explicitly matching edge features, we design a dense representation of edges to align them, bridging the direct methods and the feature-based methods in tracking. Image alignment and feature matching are performed in a general framework including both pixels and salient visual landmarks.

To track the camera pose, every new frame $\mathcal{F}_{c}$ is aligned to a reference frame $\mathcal{F}_{r}$ which is a carefully selected keyframe. First, the visual edges are extracted in $\mathcal{F}_{c}$ and $\mathcal{F}_{r}$. Then, error caused by camera pose at $\mathcal{F}_{c}$ is estimated: nonedge points $p$ in $\mathcal{F}_{r}$ are reprojected to $\mathcal{F}_{c}$ using $\omega(p, d, \xi)$ followed by the computation of photometric error; meanwhile, edge points $e_{r}$ in $\mathcal{F}_{r}$ are reprojected to a distance field derived from edges in $\mathcal{F}_{c}$ holding the minimal distance to the nearest edge point per pixel. The bottom picture of middle column in Fig. 1 illustrates a distance field, whose intensity reflects the value of distance field: whiter regions are further to edges. By multiplying a weight $\alpha$, we combine these two types of error and formulate an energy function. We apply Levenberg-Marquardt algorithm to minimize the proposed non-convex objective function.

Evaluations on real-world benchmark datasets show that our method achieves competitive results in indoor scenes. Especially, it outperform$s$ the state-of-the-art algorithms in texture-less scenes.

[1] J. Engel, T. Schops, and D. Cremers. LSD-SLAM: Large-scale direct monocular SLAM. In Proceedings of ECCV, pages 834-849, 2014.

[2] K. Hirose and H. Saito. Fast line description for line-based SLAM. In Proceedings of BMVC, 2012.

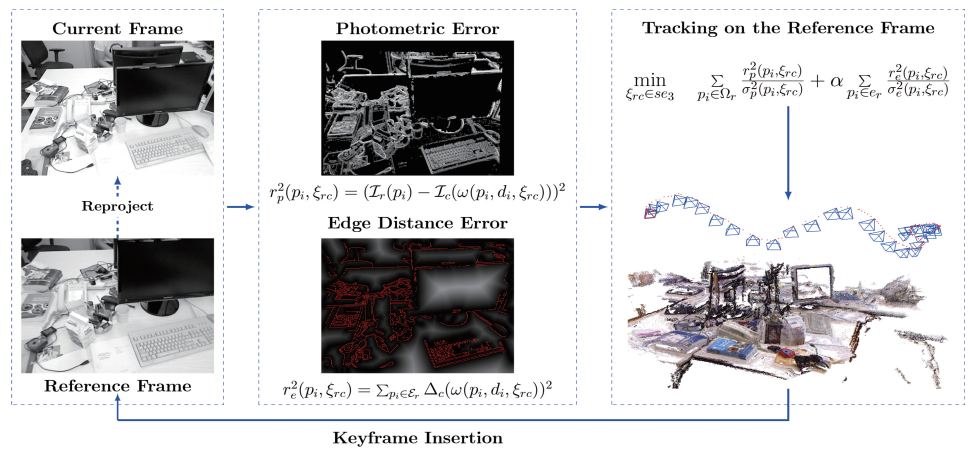

Figure 1: Overview of our approach 